\title{
Biobibliographische Notizen
}

Für zusätzliche Information s. Sherman, Joseph, 2007: Writers in Yiddish. Detroit: Thomson Gale; The YIVO Encyclopedia of Jews in Eastern Europe. <https:// yivoencyclopedia.org/>; auf Jiddisch: Leksikon fun der nayer yidisher literatur 1956-198I. In 8 Bänden. New York: Alveltlekher yidisher kultur-kongres.

Moyshe Altman (I89o Lipcani - 198I Czernowitz), Dichter, Erzähler, Essayist, erhielt eine traditionell-jüdische Bildung, besuchte ein russisches Gymnasium und erlernte im Selbststudium mehrere Sprachen. Seit 1919 in Czernowitz lebend veröffentlichte er 1920 [I9II, YIVO-Enc.] seine ersten Gedichte in Di frayhayt und war als Vortragender für die jiddische Kulturförderation von Rumänien tätig. I93I in Bukarest, während des Zweiten Weltkriegs in Zentralasien, 1945 Rückkehr nach Czernowitz. Im Zuge der stalinistischen Säuberungen 1949 zur Zwangsarbeit in Sibirien verurteilt. Seiner Freilassung 1953 folgte die erneute Rückkehr nach Czernowitz.

Iyev on khaveyrim (S. 33)

Der vortsl (S. 4I)

Bibliographie (Auswahl): Gal-Ed, Efrat, 2016: »Mojsche Altman «. In: Dies.: Niemandssprache. Itzik Manger - ein europäischer Dichter. Berlin: Jüdischer Verlag im Suhrkamp Verlag, I50-154. Rapoport, Yoyshue, 1937: "Moyshe altman als dertseyler «. In: Di tsukunft (New York) 42 (3): 184-I86. Shvarts, Itsik, I935: »Moyshe altman: >di viner karete` un andere dertseylungen «. In: Tshernovitser bleter (Czernowitz) 7 (2II), 25.3. 1935: 3. Shvarts, Itsik, I935: "Moyshe altmans dertseylungen «. In: Literarishe bleter (Warschau) I2 (I3), 29.3. 1935: 204 .

Sholem Asch (1880 Kutno - 1957 London), Prosaschriftsteller und Dramatiker, neben der traditionell-jüdischen Bildung erschloss er sich im Selbststudium die europäische Literatur und begann Hebräisch und Jiddisch zu schreiben; 1900 Veröffentlichung seiner ersten jiddischen Erzählung "Moyshele«. Zeitlebens unternahm Asch zahlreiche Reisen; er verbrachte viele Jahre in den USA und schilderte in seinen Werken das Leben der dortigen jüdischen Migranten (z. B. Onkl mozes, 1918). Aufsehen erregte in den 1930er und 1940er Jahren seine Christus-Trilogie (Der man fun natseres, Der apostol, Meri).

Iber der grenets (S. 75). Erstdruck: Der fraynd (Sankt Petersburg) I (I6), 23. I. [5. 2.] 1903: 2-3; (17) 24.I. [6.2.] 1903: 2-3; Ash, Sholem, 1903: In a shlekhter tsayt. Warschau: I. Halter et komp., 3-9. 
Oykh a mame (S. 82). Erstdruck: Ash, Sholem, 1903: In a shlekhter tsayt. Warschau: I. Halter et komp., 68-78. Übersetzungen: "A Scholar's Mother" in: Yiddish tales. I9I2, Philadelphia, Pa.: The Jewish Publication Society of America, 5I4-528 (übersetzt von Helena Frank); "Eine Mutter « in: Asch, Sholem, 2002: Zwischen den Wänden: jiddische Erzählungen zwischen Liebe und Glaube. Kassel: AQUINarte Literatur- \& Kunstpresse, 87-IOI (übersetzt von Angelika Glau).

Bibliographie (Auswahl): Ash, Sholem, I9II: Ertselungen. New York: Forverts. Bal-makhshoves, 1903: "Sholem ash: >in a shlekhter tsayt«". In: Der fraynd (Sankt Petersburg) I (202), 8. [2I.] 9. I903: I7f. Margolisov, Leo, 1904: "Sh. ash. In a shlekhter tsayt". In: Reyzen, Avrom (Hg.): Yor-bukh "progres«. Warschau, IIo-II8. Siegel, Ben, I976: The Controversial Sholem Asch: An Introduction to His Fiction. Bowling Green, Ohio: Bowling Green University Popular Press. Stahl, Nanette (Hg.), 2004: Sholem Asch Reconsidered. New Haven, Conn.: Beinecke Rare Book and Manuscript Library.

Mikha-Yoysef Berdyczewski (Bin-Gorion) (I865 Międzybóż - I92I Berlin), Schriftsteller und Denker, tätig in Hebräisch, Jiddisch, Deutsch. Besuch des Cheder, Studium an der Volozhiner Jeschiwa sowie den Universitäten von Breslau, Berlin und Bern. I886 Veröffentlichung erster Artikel in der hebräischen Presse; seit 1902 wieder in Breslau ansässig, wandte sich Berdyczewski dem Jiddischen zu, zwischen 1902 und 1907 entstanden I80 Erzählungen. Von seinen deutschsprachigen veröffentlichten Schriften sind insbesondere die Sammlungen Die Sagen der Juden und Der Born Judas zu nennen (übersetzt von Rahel Ramberg-Berdyczewsky).

Di fir shtekns (S. I05). Erstdruck: Der tog (Sankt Petersburg) I (93), 23. 4. I904: $2 \mathrm{f}$ und (94) 25.4. 1904: 2; Bin-Gorion, Micha Josef: Yidishe ksovim fun a vaytn korev. Hg. von Shmuel Werses. Jerusalem: The Magnes Press, The Hebrew University, 57-64.

Der kadish (S. II2)

Bibliographie (Auswahl): Fridman, Dovid-Arye, I924: "Mikhe-yoysef berditshevskis zeks bender yidishe ksovim«. In: Di tsukunft (New York) 29 (Iо): 626-629. K-s, B. [Karlinski, Ber], I925: "Der vayter korev«. In: Der moment (Warschau) I6 (26), 30. I. 1925: 8; (38) I3. 2. 1925: 8. Lev, Simkhe, I966: "M. y. berditshevski der dertseyler oyf hebreish un yidish «. In: Svive (New York) I9: 2I-28. Mayzl, Nakhmen, I924: "M. y. berditshevki in yidish «. In: Literarishe bleter (Warschau) I (2), I6. 5. I924: 3. Werses, Shmuel, I98I: "M. J. Berdyczewski as a Yiddish Writer«. In: Bin-Gorion, Micha Josef: Yidishe ksovim fun a vaytn korev. Hg. von Shmuel Werses. Jerusalem: The Magnes Press, The Hebrew University, V-LVII (siehe auch Bibliographie auf Seite סב). 
Dovid Bergelson (I884 Okhrimovo - 1952 Moskau), Prosaschriftsteller und Dramatiker, erhielt neben der religiösen auch eine säkulare Bildung. Erste schriftstellerische Versuche auf Hebräisch, 1909 Veröffentlichung der jiddischen Erzählung "Arum vokzal". In Kiew lebend engagierte er sich seit ihrer Gründung 1918 in der Kultur-lige, zog 192I für einige Jahre nach Berlin, bevor er 1934 in die Sowjetunion übersiedelte. Als Mitglied des Jüdischen Antifaschistischen Komitees wurde Bergelson am I2. August 1952 erschossen.

On a nomen (S. 93). Erstdruck: Laut eigenen Angaben entstand die Erzählung 1909-1910 und wurde 1917-1918 überarbeitet (»Materyaln tsu bergelsons bio-bibliografye«. In: Visnshaft un revolutsye $(\mathrm{I}-2)$, 1934, Kiew: Farlag fun der alukrainisher visnshaftlekher akademye: 67-73, hier 67). Erste Publikation in: Naye tsayt (Kiew) (29), 14. [27.]. I0. 1917: 2f. Übersetzungen: "Without a Name« in: Neugroschel, Joachim (Hg., Übers.), 2002: No Star Too Beautiful: Yiddish Stories from 1382 to the Present. New York, NY: W. W. Norton \& Company, 418-424.

Frayndshaft (S. 99)

Bibliographie (Auswahl): Estraikh, Gennady, 2005: In Harness: Yiddish Writers' Romance With Communism. Syracuse, NY: Syracuse University Press. Murav, Harriet, 20I2: "Marking Time. Bergelson and Bergson «. In: Aptroot, Marion, Efrat Gal-Ed, Roland Gruschka und Simon Neuberg (Hg.): Leket: yidishe shtudyes haynt. Düsseldorf: düsseldorf university press, I53-I7I. Murav, Harriet, 2019: David Bergelson's Strange New World. Untimeliness and Futurity. Bloomington, Ind.: Indiana University Press. Schachter, Allison, 2008: "Bergelson and the Landscape of Yiddish Modernism«. In: East European Jewish Affairs 38 (I): 7-19. Sherman, Joseph und Gennady Estraikh (Hg.), 2007: David Bergelson: From Modernism to Socialist Realism. London: Legenda.

Der Nister - > Der Verborgene` (Pinkhes Kahanovitsh) (I884 Berditschew - I950 Moskau), Dichter und Prosaschriftsteller, erhielt eine traditionell-jüdische Bildung, las daneben auch säkulare Literatur und begann hebräische Texte zu schreiben. 1907 literarisches Debüt auf Jiddisch mit dem Band Gedanken un motivn: lider in proze; seit 1918 in Kiew, Engagement in der Kultur-lige, von I92I bis 1926 in Berlin und Hamburg, anschließend Übersiedelung in die Sowjetunion. Als Mitglied des Jüdischen Antifaschistischen Komitees wurde er 1949 Opfer der sowjetischen Säuberungen und starb 1950 in einem Lagerkrankenhaus.

In vaynkeler (S. II7). Erstdruck: Parallel in Dos naye leben (New York) I (I), I. IO. 1922: 4-19 und Shtrom (Moskau) I (2), I922: 9-25. Übersetzungen: "In the Wine Cellar" in: Neugroschel, Joachim (Hg., Übers.), 1987: Great Tales of Jewish Phantasy and the Occult. New York, NY: Overlook, 246-264; 
"Im Weinkeller« in: Mantowan-Kromer, Daniela (Hg.), I991: Unterm Zaun. Jiddische Erzählungen. Frankfurt am Main: Insel, I34-I55 (übersetzt von Martina Eicheldinger).

Geyendik (S. 134). Erstdruck: Unter dem Titel "Er un ikh « in: Dorem-afrike (Johannesburg) I, I922: 16-23.

Bibliographie (Auswahl): Bechtel, Delphine, I990: Der Nister's Work 1907-1929: A Study of a Yiddish Symbolist. Berne u. a.: Lang. Estraikh, Gennady (Hg.), 20I4: Uncovering the Hidden: The Works and Life of Der Nister. London: Legenda. Krutikov, Mikhail, 2019: Der Nister's Soviet Years. Yiddish Writer as Witness to the People. Bloomington, Ind.: Indiana University Press. Mayzl, Nakhmen, 1924: "Der nister (tsum dershaynen fun tsvey bender 'gedakht`, yidisher literarisher farlag, berlin 1923)«. In: Literarishe bleter (Warschau) i (I8), 5.9.1924: 2. Mantovan, Daniela, 1993: Der Nister and His Symbolist Short Stories (I9I3-I929): Patterns of Imagination. New York, NY: Columbia University. Tseytlin, Arn, I924: »Der nister: 'gedakht««. In: Bikher-velt (Warschau) 3 (I-2): I7-I9.

Dovid Frishman (I859 Zgierz - 1922 Berlin), hebräischer und jiddischer Schriftsteller, Übersetzer, Literaturkritiker, Herausgeber; erhielt neben einer jüdischen auch eine säkulare Bildung; I878 Veröffentlichung seiner ersten hebräischen Erzählung. In Berlin und Warschau verdiente er seinen Lebensunterhalt mit Übersetzungen aus dem Deutschen und als Verfasser literarischer Essays, I886 Herausgeber der Tageszeitung Ha-yom. I888 Veröffentlichung seines ersten jiddischen Gedichts "Afn bergl «; nach einem Studium in Breslau I895 Rückkehr nach Warschau, wo er neben seiner schriftstellerischen Arbeit auch als Übersetzer und Herausgeber tätig war.

Der tants (S. 34I). Erstdruck: Teater-velt (Warschau) 2 (6), I909: II-I4. Vegn eyn eyntsik pintele (S. 347)

Bibliographie (Auswahl): Mayzl, Nakhmen, I930: "Shoyn lang tsayt! (tsu der oysgabe fun ale verk fun dovid frishman in idish un hebreish)«. In: Haynt (Warschau) 23 (9I), I8. 4. I930: 9. Niger, Shmuel, I922: "Dovid frishman - der shriftshteler «. In: Dos naye leben (New York) I (I), Oktober: 58-63. Parush, Iris, 2015: "Narodowość, syjonizm i dyskurs teologiczny: Dawid Fryszman wobec Chaima Nachmana Bialika«. In: Studia Judaica I (35): 55-84. Tseytlin, H[ilel], I909: "Dovid frishman (etlikhe verter vegen zayne ertsehlungen) «. In: Haynt (Warschau) 2 (86), I5. [28.] 4. I909: 2f; (9I) 2I. 4. [4. 5.] I909: $2 \mathrm{f}$.

Avrom-Moyshe Fuks (Fuchs) (I890 Jezierna - I974 Tel Aviv), Prosaschriftsteller, Journalist, erhielt neben der religiösen auch eine säkulare Schulbildung. I9II erschien seine erste Erzählung "Bilder funem shtetl«. In verschiedenen 
Städten Galiziens lebend engagierte er sich in der sozialistischen Bewegung, gehörte der Lemberger Schriftstellergruppe Yung-Galitsye an, bevor er I9I2 in die USA übersiedelte. Zwei Jahre später Rückkehr nach Wien, Korrespondent verschiedener Zeitungen; nach seiner Verhaftung 1938 gelangte Fuks über die Schweiz und Frankreich nach England, I950 emigrierte er nach Israel.

Bay tog (S. 329)

Der alter koyekh (S. 336)

Bibliographie (Auswahl): Ayzland, Ruvn, 1925: A. m. fuks". In: Der inzl (New York) (2): 5- II. Gros-Tsimerman, M[oyshe], I962: "Shver tsu shraybn shver (vegn > di nakht un der tog fun a. m. fuks) «. In: Di goldene keyt (Tel Aviv) 42: 69-76. Karlinius, Ber, 1925: "Literarishe skitsen. Der mentsh fun fleysh un blut «. In: Der Moment (Warschau) I6 (63), I5.3. 1925: 4. Müller, Karl, 2003: "Aspekte jiddischer Prosa am Beispiel von Abraham Mosche Fuchs«. In: Eidherr, Armin (Hg.): Jiddische Kultur und Literatur aus Österreich. Zwischenwelt. Bd. 8. Klagenfurt: Drava, I67-184. Niger, Shmuel, I925: "A. m. fuks - a beyzer talant «. In: Literarishe bleter (Warschau) 2 (52), 30. 4. 1925: 6. Perle, Y., I925: "Nakete proze«. In: Undzer folkstsaytung (Warschau) 3 (I4), I6. I. I925: 5 .

Yekhiel Hofer (1906 Warschau - 1972 Yafo), Erzähler, Dichter, Essayist, erhielt vor seinem Medizinstudium eine traditionell-jüdische Bildung. Während seiner Tätigkeit bei einer jüdischen Wohlfahrtseinrichtung in Warschau begann er auf Polnisch zu schreiben, wandte sich dann aber der jiddischen Sprache zu. Den Zweiten Weltkrieg überlebte er in der Sowjetunion, I948 zog er nach Paris, I95I nach Israel. Hofers Werke erschienen zahlreich in der jiddischen Presse Polens, Frankreichs und Israels. Noch in Paris erschien I950 sein Gedichtband Lider fun der nakht, 1963 dann in Tel Aviv der Erzählungsband Amol und 1966 Reb tankhem.

Reb tankhem [Einführung] (S. I4I)

Der toyt fun reb tankhemen (S. I46)

Bibliographie (Auswahl): Gros-Tsimerman, Moyshe, 1973: "Yekhiel hofer ". In: Di goldene keyt (Tel Aviv) 78: I46f. Hirshhoyt, Yekhiel, 1973: "Yekhiel hofer - der dertseyler fun yidisher ashires«. In: Di tsukunft (New York) 79 (2): 5I-54. Yanasovitsh, Yitskhok, 1974: "Der shrayber yekhiel hofer«. In: Di goldene keyt (Tel Aviv) 82: 150-160. Goldberg, Ruvn, 1986: „Yekhiel Hofer «. In: Kagan, Berl (Hg.): Leksikon fun yidish shraybers. New York, Sp. 2IO-2I2 (siehe auch Bibliographie in Sp. 212).

Ber Horovits (Horowitz) (1895 Maydan - 1942 Ostgalizien), Schriftsteller und Maler, schloss 1914 das polnische Gymnasium in Stanisławów ab, erhielt da- 
neben eine traditionell-jüdische Bildung durch Privatlehrer und studierte Medizin in Wien. I918 erste Gedichtveröffentlichungen in der Wiener Zeitschrift Nayland; in den folgenden Jahren unternahm Horovits zahlreiche Reisen, trat auch als Übersetzer und Illustrator (Vunderlekhe mayses, 1923) in Erscheinung. Über seinen gewaltsamen Tod 1942 gibt es keine zuverlässigen Angaben.

Di legende fun der madonna (S. 159). Erstdruck: Vunderlekhe mayses. Warschau: Vanderer, 1923, 69-9r. Übersetzungen: "The Legend of the Madonna" in: Neugroschel, Joachim (Hg., Übers.), 1987: Great Tales of Jewish Fantasy and the Occult. New York, NY: The Overlook Press, 448-459.

Der gilgl (S. I7I). Erstdruck: Vunderlekhe mayses. Warschau: Vanderer, 1923, $43-67$.

Bibliographie (Auswahl): Gal-Ed, Efrat, 2016: Niemandssprache. Itzik Manger - ein europäischer Dichter. Berlin: Jüdischer Verlag im Suhrkamp Verlag, 197, 203-206. Mark, Arn, 1930: "Ber horovits". In: Literarishe bleter (Warschau) 7 (22), 30.5.1930: 404-407. Perle, Yehoyshue, 1926: "Tsu gast in kroke«. In: Literarishe bleter (Warschau) 3 (130), 29. I0. 1926: IIf. Tseytlin, Arn, 1924: »[Retsenzye vegn:] dov-ber horovits: ,vunderlekhe mayses «. In: Bikher-velt (Kiew) 3 (I-2): 25-27. Vaynig, Naftole, 1924: "Ber horovits ". In: Literarishe bleter (Warschau) I (5), 6.6.1924: 4. Vaynper, Tsishe, I954: "Ber horovits". In: Yidishe kultur (New York) I6 (II): 23-26.

Efroim Kaganovski (Kaganowski) (1893 Warschau - 1958 Paris), Prosaschriftsteller, besuchte eine Kunstschule und veröffentlichte 1909 seine erste Erzählung "Bay nakht in dorf « in Di teater velt. I9I4 Flucht nach Odessa, I92I Rückkehr nach Warschau, Veröffentlichung weiterer Erzählungen und zahlreicher Beiträge für verschiedene Zeitungen und Zeitschriften. Den Zweiten Weltkrieg überlebte Kaganowski in Usbekistan, bevor er sich 1949 in Paris niederließ, wo u. a. eine neue Ausgabe seiner Erzählungen mit eigenen Zeichnungen erschien.

Meylekhl kilke (S. 35I). Erstdruck: Haynt (Warschau) 28 (IOI), 3. 5. 1935: 7 (mit Abweichungen).

Der ,khokhem ( (S. 35I). Erstdruck: Kaganovski, Efroim, 1937: Figurn. Warschau: Pen-bikher, 27-37. Übersetzungen: "The Wise One« in: Kaganovski, Ephraim, 2016: Jewish Warsaw between the Wars. Lexington: CreateSpace Independent Publishing Platform, 39-50 (übersetzt von Bracha B. Weingrod). Bibliographie (Auswahl): Finkelshteyn, Leo, 1932: „Vegn e. kaganovskis noveln «. In: Literarishe bleter (Warschau) 9 (29), 15.7.1932: 462. Kaganovski, Efroim, 1949: Yidishe shrayber in der heym. Łódź: Yidish-bukh. Mayzl, Nakhmen, 1938: "Efroim kaganovskis bukh >figurn««. In: Literarishe bleter (Warschau) I5 (2), 7. I. 1938: Igf. Shimel, Moyshe, 1937: "Efroim kaganovski. Tsum dershaynen fun zayn noveln-bukh: , figurn` (shtrikhn tsu a por- 
tret)«. In: Haynt (Warschau) 30 (2II), I0.9. 1937: 7. Yakubovitsh, AvromLeyb, I92I: "Literarishe geshprekhn XXXIII. A zamlung fun shikzaln «. In: Haynt (Warschau) I4 (29I), 23. I2. 1937: 3.

Alter-Sholem Katsizne (Kacyzne) (I885 Wilna - I94I Tarnopol), Schriftsteller und Photograph, besuchte neben dem Cheder eine russischsprachige Volksschule, las als Autodidakt Literatur in mehreren Sprachen, bevor er sich in Ekaterinoslav als Photograph ausbilden ließ. Nach zwei russischen Erzählungen veröffentlichte er I9I5 anlässlich des Todes von Y. L. Perets Erinnerungen an seinen literarischen Mentor auf Jiddisch. Im Zusammenhang mit einem Auftrag, das polnische Judentum photographisch zu dokumentieren, unternahm Katsizne zahlreiche Reisen. Nach Ausbruch des Zweiten Weltkriegs floh er nach Tarnopol, wo er am 7. Juli i94I ermordet wurde.

A terk (S. 363). Erstdruck: Ringen (Warschau), Nr. I, I92I: 6-I4.

Bibliographie (Auswahl): Emyot, Yisroel, 1968: "Di gezamlte shriftn fun alter katsizne«. In: Di tsukunft (New York) 30 (7-8): 37If. Grudberg-Turkov, Yitskhok-Ber, 1967: „Der peretsyaner alter katsizne. Alter katsizne, ’gezamlte shriftn««. In: Di goldene keyt (Tel Aviv) 6r: 2IO-2I3. Kacyzne, Alter, 1999: Poyln. Eine untergegangene Welt. Hg. von Marek Web. Berlin: AufbauVerlag. Katsizne, Alter, 1967-1972: Gezamlte shriftn. In 4 Bänden. Tel Aviv: Farlag y. 1. perets. Litvin, Mortkhe, 1977: "Alter katsizne - der dikhter«. In: Di tsukunft (New York) 83 (2): 64-66. Mark, B., 1985: "Alter katsizne. lebn un verk«. In: Yidishe kultur (New York) 47 (5/6): 46-49. Rotbaum, Yankev, 1982: "Alter katsizne - der mentsh un kinstler«. In: Yidishe kultur (New York) 44 (2): 6-I4 und (3): 25-30.

Rokhl Korn (I898 Podliski - I982 Montreal), Dichterin, Prosaschriftstellerin, trat zunächst mit polnischen Kurzgeschichten in Erscheinung. Unter dem Eindruck des Ersten Weltkriegs und der Pogrome wandte sie sich der jiddischen Sprache zu, die sie erst erlernen musste; ihr erstes jiddisches Gedicht erschien I9I9 im Lemberger togblat. In der Zwischenkriegszeit veröffentlichte sie zahlreiche Gedichte, Erzählungen und Essays; 194I floh Korn mit ihrer Tochter nach Usbekistan, 1949 emigrierte sie nach Kanada.

Der letster veg (S. 37I). Übersetzungen: "The Road of No Return « in: Forman, Frieda, Ethel Raicus, Sarah Silberstein Schwartz und Margie Wolfe (Hg.), 1994: Found Treasures: Stories by Yiddish Women Writers. Toronto: Second Story Press, 2II-222 (übersetzt von Miriam Waddington); [dieselbe Übersetzung] in: Raphael, Linda Schermer und Marc Lee Raphael (Hg.), I999: When Night Fell: An Anthology of Holocaust Short Stories. New Brunswick, NJ und London: Rutgers University Press, I9I-I98; „Der letzte Weg“ in: Forman, Frieda, Ethel Raicus, Sarah Silberstein Schwartz und Margie Wolfe 
(Hg.), 1999: Aus der Finsternis geborgen: Erzählungen jiddischer Autorinnen. Salzburg: Müller, 167-178 (übersetzt von Armin Eidherr); "The End of the Road « in: Neugroschel, Joachim (Hg., Übers.), 2002: No Star Too beautiful: Yiddish Stories from I382 to the Present. New York, NY, Norton, 633-64I. Mayn heym un ikh (S. 380)

Bibliographie (Auswahl): Goldfrad, Keren, 2oII: "At the Crossroads: Between >Der Letster Veg` and the Road of No Return «. In: Prism (3): I2-I7. Mayzl, Nakhmen, I935: "Oyf poylisher roy-erd «. In: Haynt (Warschau) 28 (264), I5. II. 1935: 7. Mukdoni, Aleksander, 1958: "Yidish dorf un yidishe shtot «. In: Di tsukunft (New York) 63 (4): 199-202. Oyerbakh, Rokhl, 1978: "Rokhl korn. Portret, varshe 1933". In: Di tsukunft (New York) 84 (I): 20-22. Seelig, Rachel, 20I4: "Like a Barren Sheet of Paper: Rokhl Korn from Galician Orchards to Postwar Montreal «. In: Prooftexts 34 (3): $349-377$.

(Hinde) Ester Kreytman (1892 Biłgoraj - I954 London), Prosaschriftstellerin, Übersetzerin. Die Schwester von Yisroel-Yoyshue und Yitskhok Bashevis Zinger erhielt keinerlei formale Bildung, erlernte im Selbststudium mehrere Sprachen und erwarb so umfassende Literaturkenntnisse. Seit 1912 in Belgien, später in England lebend kehrte sie immer wieder nach Warschau zurück, wo sie im Schriftstellerverband tätig war. Kreytman übersetzte u.a. Dickens' A Christmas Carol ins Jiddische und veröffentlichte 1936 den autobiographischen Roman Der sheydim-tants; darauf folgten der Roman Brilyantn (1944) und der Erzählband Yikhes (1954).

Di naye velt (S. 413). Übersetzungen: »The New World « in: Yale Review 73 (Summer 1984): 525-532 (übersetzt von Joshua A. Fogel); "The New World" in: Lilith I6 (2), (Frühling I99I): IO-I2 (übersetzt von Barbara Harshav), nachgedruckt in: Frieda Forman, Ethel Raicus, Sarah Silberstein Schwartz und Margie Wolfe (Hg.), 1994: Found Treasures: Stories by Yiddish Women Writers. Toronto: Second Story Press, 77-84; "Die neue Welt « in: Forman, Frieda, Ethel Raicus, Sarah Silberstein Schwartz und Margie Wolfe (Hg.), 1999: Aus der Finsternis geborgen: Erzählungen jiddischer Autorinnen. Salzburg, Wien: Müller, 27-33 (übersetzt von Armin Eidherr); Sylvia Paskin (Hg.), 2004: Blitz and Other Stories. Jefferson, NC: McFarland \& Company, Inc. Publishers (übersetzt von Dorothee van Tendeloo).

Tsvey bibliotekn (S. 419)

Bibliographie (Auswahl): Garfinkel, Lea, 2008: "Ester Kreitman - A Scion of the Singer Family: The Rejected Sister: with Hebrew Translation of her Story , The New World «. In: Tova Cohen (Hg.): Nashim be-tarbut yidish. Women in Yiddish Culture. Ramat-Gan: University Bar-Ilan, 175-182. Jones, Faith, 2004: "An Ester Kreytman [Esther Kreitman] Bibliography «. In: The Mendele Review: Yiddish Literature and Language, I. 9. 2004, <http://yiddish. 
haifa.ac.il/tmr/tmro8/tmro80o9.htm.> 15.9.2020. Norich, Anita, 1990: »The Family Singer and the Autobiographical Imagination«. In: Prooftexts IO (I): 9I-I07. Wise, Yaakov, 2015: "The First Jewish Feminist?» In: Jewish Quarterly62(I): 44-47.

Moyshe Kulbak (1896 Smorgon - I937 Minsk), Dichter, Prosaschriftsteller, Dramatiker, erhielt neben einer umfassenden jüdischen auch eine säkulare Bildung. Er schrieb Hebräisch, bevor er 1916 sein erstes jiddisches Gedicht "Shterndl « veröffentlichte. Neben seiner Tätigkeit als Lehrer in Minsk und Wilna engagierte sich Kulbak in den jiddischen Kulturinstitutionen. 1920 Emigration nach Berlin, 1928 Rückkehr nach Minsk. Im Zuge der stalinistischen Repressionen wurde er 1937 nach einem Schauprozess erschossen, 1956 postum rehabilitiert.

Munye der foygl-hendler un malkele zayn vayb (S. 403). Erstdruck: Di yidishe velt (Wilna) (6), (September 1928): 333-346. Übersetzungen: »Munie the Bird Dealer " in: Howe, Irwing und Eliezer Greenberg (Hg.), I955: A Treasury of Yiddish Stories. London: Andre Deutsch, 342-350 (übersetzt von Norbert Guterman); "Muni The Bird-Seller« in: Leftwich, Joseph (Hg., Übers.), 1974: An Anthology of Modern Yiddish Literature. The Hague (u. a.): Mouton, $85-92$.

Bibliographie (Auswahl): Finkin, Jordan, 2010: ")Like Fires in Overgrown Forests : Moyshe Kulbak's Contemporary Berlin Poetics«. In: Estraikh, Gennady und Mikhail Krutikov (Hg.): Yiddish in Weimar Berlin. At the Crossroads of Diaspora Politics and Culture. London: Legenda, 73-88. Koller, Sabine, 20I4: "Jiddische Literatur im Krieg: Moyshe Kulbak und Yisroel Rabon «. In: Diner, Dan (Hg.): Jahrbuch des Simon-Dubnow-Instituts XIII, 237-26r. Finkin, Jordan, 20I6: »Yiddish Ethnographic Poetics and Moyshe Kulbak's ,Vilne«". In: Kilcher, Andreas B. und Gabriella Safran (Hg.): Writing Jewish Culture. Paradoxes in Ethnography. Bloomington, Ind.: Indiana University Press, 94-II8. Gal-Ed, Efrat, (in Druck): „A delicious cooing and chirping: Reading the first chapter of Moyshe Kulbak's >Munye the Bird-Seller «. In: Gal-Ed, Efrat, Natasha Gordinsky, Sabine Koller und Yfaat Weiss (Hg.): In their Surroundings: Localizing Modern Jewish Literatures in Eastern Europe. Göttingen: Vandenhoeck \& Ruprecht. Seelig, Rachel, 20I6: ")A Youthful Rogue Am I : Moyshe Kulbak between Exile and Arrival «. In: Dies.: Strangers in Berlin. Modern Jewish Literature between East and West, 1919-1933. Ann Arbor, Mich.: University of Michigan Press, 79-100.

Meyer Kutshinski (1904 Włocławek - 1976 São Paulo), jiddischer Schriftsteller und Lehrer, besuchte nach dem Cheder ein polnisch-hebräisches Gymnasium; sammelte ethnographisches Material für das YIVO-Institut, war in der Linke Poale Zion aktiv. 1935 Emigration nach Brasilien, zunächst als Hau- 
sierer, später als Lehrer für jiddische Literatur tätig; mit Erzählungen und Essays in beinahe allen jiddischen Zeitungen Brasiliens vertreten, ebenso in den in New York erscheinenden Zeitschriften Di tsukunft und YIVO-bleter u. a. Daneben entstanden zwei Theaterstücke und eine Studie bezüglich der portugiesischen Elemente im Jiddischen.

Di mona liza (S. 391). Übersetzungen: "Mona Lisa« in: Kucinski, Meir, 2002: Imigrantes, mascates \& doutores. São Paolo: Ateliê Editorial, 55-62 (übersetzt ins Portugiesische von Meiri Levin).

Mayn ershte bagegenish (S. 397)

Bibliographie (Auswahl): Cytrynowicz, Roney, 2018: "The Yiddish Side of Jewish Brazil: Cultural Endeavors and Literary Heritage«. In: Chinski, Malena und Alan Astro (Hg.): Splendor, Decline, and Rediscovery of Yiddish in Latin America. Leiden, Boston: Brill, I5-4I. Emyot, Yisroel, 1965: Guts un shlekhts. Meyer kutshinski: , nusekh brazil««. In: Di tsukunft (New York) 70 (3): I36f. Falbel, Nachman, 2010: Literatura Ídiche no Brasil. São Paulo: Humanitas. Izban, Shmuel, 1965: "Meyer kutshinskis brazilyaner dertseylungen. > Nusekh brazil fun meyer kutshinski «. In: Unzer kiem (Paris) (44), (Januar 1965): 16. Rayzman, Yitskhok Z., 1975: Yidishe sheferishkeyt in lender fun portugalishn loshn. Safed, 289-296.

Malasha Mali (I92I Łódź - 2008?), Dichterin, Prosaschriftstellerin, erhielt neben einer jüdischen eine säkulare Bildung; Engagement in sozialistischzionistischen Jugendorganisationen, 1938 erste Beiträge für die Warschauer Zeitungen Bafrayung und Dos vort. Während des Zweiten Weltkriegs in Russland, 1946 Rückkehr nach Polen, von dort in die DP-Lager nach Deutschland verbracht. Veröffentlichungen in der jiddischen Presse; 1948 erschien ihr erster Erzählungsband Geviter, 1949 Emigration nach Israel. Dort schrieb sie für verschiedene Zeitungen und Zeitschriften wie Di goldene keyt, war Mitglied von Yung-Yisroel und veröffentlichte 1963 den Erzählungsband Tsvey veltn.

,Maciejkar (S. 235)

Bibliographie (Auswahl): Bikl, Shloyme, 1953: "Tsvey premirte dertseylungen «. In: Di tsukunft (New York) 58 (7): 440. Mayzl, Nakhmen, 1949: „Dray naye yidishe dertseylerins". In: Yidishe kultur (New York) II (II): 23-30. Rapoport, Yoyshe, 1964: »An interesant prozebukh «. In: Di goldene keyt (Tel Aviv) 50: 327-329. Ravitsh, Melekh, 1958: "Malasha mali«. In: Ders.: Mayn leksikon. Bd. 3, Montreal: Notern printing un steyshoneri, $246 \mathrm{f}$.

Itsik Manger (Isidor Helfer) (190I Czernowitz - 1969 Gedera), Dichter, Prosa- und Theaterschriftsteller, Essayist, besuchte nach dem Cheder für kurze Zeit ein Gymnasium, erwarb im Selbststudium umfangreiche Kenntnisse 
der Weltliteratur, schrieb erste Gedichte auf Deutsch; 192I Veröffentlichung seiner ersten jiddischen Ballade in der Zeitschrift Kultur (Czernowitz). Seit I928 immer wieder in Warschau; nach seiner erzwungenen Emigration 1938 gelangte Manger über Paris nach London, es folgten Aufenthalte in den USA und Israel.

Gele (S. 253). Erstdruck: Naye folkstsaytung (Warschau) I2 (5I), 19. 2. 1937: 6-7. A portret fun a shnaydershtub (S. 258). Erstdruck: Mit dem Untertitel ,Aus meinem Tagebuch`, in: Der veker (New York), I. 4. und I. 5. 196I. Übersetzungen: "A Portrait of a Tailor's Workshop « in: Manger, Itzik, 2002: The World According to Itzik: Selected Poetry and Prose. Hg. und übers. von Leonard Wolf. New Haven, Conn. (u. a.): Yale University Press, I23-134; "Porträt einer Schneiderstube«. In: Sinn und Form 6, 2016: 265-274 (übersetzt von Almut Seiffert).

Bibliographie (Auswahl): Bashevis, Yitskhok, 1939: "Itsik manger, 'noente geshtaltn «". In: Di tsukunft (New York) 44 (7): 425. Frakes, Jerold C., 2008: / Ella b. Moses b. Abraham the Patriarch, Verse Colophon to תפילה למשה. In: Ders.: Early Yiddish Texts II00-1750: With Introduction and Commentary. Oxford: Oxford University Press, 749 f. Gal-Ed, Efrat, 2016: Niemandssprache: Itzik Manger-ein europäischer Dichter. Berlin: Jüdischer Verlag im Suhrkamp Verlag. Hofer, Yekhiel, I962: "Itsik manger der prozaiker «. In: Di goldene keyt (Tel Aviv) 42: 79-88. Mukdoni, Aleksander, 1938: " Noente geshtaltn ‘ fun itsik manger «. In: Undzer tog (Wilna) 20 (300), 30. I2. 1938: 5. Turniansky, Chava, I999: "Mejdlech in der altjidischer literatur «. In: Röll, Walter und Simon Neuberg (Hg.): Jiddische Philologie. Festschrift für Erika Timm. Tübingen: Max Niemeyer Verlag, $7^{*}-20^{*}$.

Anna Margolin (Roza Lebensboym) (1887 Brest - I952 New York), Dichterin, Journalistin, besuchte ein russisches Gymnasium in Odessa und erhielt Privatunterricht, 1906 erstmals nach New York. Zunächst als Sekretärin von Khayim Zhitlovski tätig, veröffentlichte sie 1909 eigene Kurzgeschichten in Fraye arbeter-shtime. Nach einigen Jahren in Europa und einem kurzen Aufenthalt in Palästina I9I4 Rückkehr nach New York, wo sie u. a. für die Rubrik »In der froyen velt « der Zeitung Der tog schrieb. I92I erschienen ihre ersten Gedichte unter dem Pseudonym >Anna Margolin`, I929 Veröffentlichung ihres einzigen Gedichtbands Lider.

Oyf a bal (S. 277). Erstdruck: unter dem Pseudonym Khane Barit, in: Fraye arbeter shtime (New York), II. 7. 1914: 3.

Fun a tagebukh (S. 269). Erstdruck: unter dem Pseudonym Khave Gross, in: Di tsukunft (New York) I4 (4), (1909): 243-247.

Bibliographie (Auswahl): Iceland, Reuben, 1954: Fun undzer friling. New York: Farlag indzl. Kumove, Shirley, 1998: "Drunk from the Bitter Truth: 
The Life, Times and Poetry of Anna Margolin «. In: Swartz, Sarah und Margie Wolfe (Hg.): From Memory to Transformation: Jewish Women's Voices. Toronto: Second Story Press, 35-48. Manger, Itzik, I980: "Ana margolin «. In: Ders.: Shriftn in proze. Tel Aviv: Farlag y.l. perets, 249-253. Novershtern, Avraham, I991: "Ana margolin: materyaln tsu ir poetisher geshtalt». In: YIVO bleter, new series, I: I29-I7I. Ders., I991: ") Who Would Have Believed That a Bronze Statue Can Weep : The Poetry of Anna Margolin«. In: Margolin, Anna: Lider. Poems (zweisprachig). Jerusalem: The Hebrew University, V-LVIII (übersetzt von Robert Wolf). Sigal, Y. Y., I952: "Ana margolin «. In: Di tsukunft (New York) 57 (8): 349-35I.

Kadye Molodovski (Kadia Molodowsky) (I894 Bereza Kartuska - I975 Philadelphia), Schriftstellerin, Herausgeberin, Lehrerin, erhielt sowohl eine religiöse als auch eine säkulare Bildung. Nach Abschluss des Lehrerseminars u. a. in Warschau, Odessa und Kiew als Lehrerin tätig; I920 erste Gedichtveröffentlichungen in Eygns. Neben zahlreichen Publikationen (Gedichte, Erzählungen, Kinderliteratur, Essays) engagierte sich Molodovski im jiddischen Schriftstellerverband. I935 emigrierte sie in die USA, die Jahre 1950-52 verbrachte sie in Tel Aviv.

Di ferte mitsve (Aus dem Zyklus "Tulye shor dertseylt «) (S. 223). Erstdruck: $A$ shtub mit zibn fentster. New York: Farlag matones, 1957, 70-75, darin auf Mai 1943 datiert.

Frayndshaft (S. 227). Erstdruck: Ibid., 217-219, darin auf 1946 datiert.

In a living-rum (S. 230). Erstdruck: Ibid., 95-98, darin auf I3. Juli I947 datiert. Alle Kurzgeschichten übersetzt in: A House with Seven Windows. Syracuse, NY: Syracuse University Press, 70-74, 227-229 und 99-IO2 (übersetzt von Leah Schoolnik).

Bibliographie (Auswahl): Gonshor, Anna, 1997: Kadye Molodowsky in Literarishe bleter, 1925-35: Annotated Bibliography. MA Thesis, Montreal: McGill University. Jonas-Märtin, Esther, 2008: "Zwischen Journalismus und Belletristik: Kadye Molodovsky (I894-I974)«. In: Lappin, Eleonore und Michael Nagel (Hg.): Deutsch-jüdische Presse und jüdische Geschichte. Bd. I. Bremen: Edition Lumière, 375-386. Klepfisz, Irena, I994: „Di Mames, Dos Loshn/The Mothers, the Language: Feminism, Yidishkayt, and the Politics of Memory«. In: Bridges 4 (I): I2-47. Kope-Lovitsh, Rivke, I972: "Di dikhterin un prozaikerin kadye molodovski«. In: Almanakh (Paris) 3 , I972: 33I-338. Pareigis, Christina, 20I6: "Sprache und Überleben. Jiddisch in Amerika. Kadya Molodowsky (1894-1975) «. In: Bischoff, Doerte (Hg.): Exil-Literatur - Judentum. München: Edition text + kritik, 195-216. Rapoport, Yehoyshue, 1964: "A bukh mit zibn mayles... kadye molodovski: ‘a shtub mit zibn fentster»". In: Zayn (New York) io (36), Januar I964: I0-I5. 
Hersh-Dovid Nomberg (1876 Mszczonów - 1927 Otwóck), hebräischer und jiddischer Essayist, Prosaschriftsteller, politischer und sozialer Aktivist, erhielt neben einer traditionell-jüdischen Erziehung auch eine säkulare Bildung, erwarb im Selbststudium umfassende Sprachkenntnisse; 1900 Veröffentlichung seines ersten jiddischen Gedichts "Der novi «. In den folgenden Jahren schrieb Nomberg für die jiddische und hebräische Presse und unternahm zahlreiche Reisen. Neben seinen politischen Aktivitäten, u.a. als Abgeordneter des Sejms, engagierte sich Nomberg im jiddischen Schriftstellerverband, dessen Vorsitzender er von 1925 bis 1927 war.

Shvayg, shvester! (S. 26I). Übersetzungen: "Don't Say a Word!« in: Nomberg, Hersh David, 2019: Warsaw Stories. Amherst, Massachusetts: White Goat Press, I40-I46 (übersetzt von Daniel Kennedy).

Bibliographie (Auswahl): Hadda, Janet, 1988: Passionate Women, Passive men. Suicide in Yiddish Literature. New York: State University of New York Press, Albany. Mayzl, Nakhmen, 1937: "H.d. nomberg«. In: Haynt (Warschau) 30 (266), 19. II. 1937: 7. Shtern, Yisroel, 1937: "Nombergisher stil«. In: Haynt (Warschau) 30 (266), 19. II. 1937: 7, Io. Vayntroyb, Yitskhok, I928: "Nomberg's literarishe yerushe. Vos iz biz itst shoyn gezamelt gevoren un vos vert vayter gezamelt? "In: Der moment (Warschau) I9 (279), 2. I2. 1928: 5 .

Yoysef Opatoshu (Yoysef-Meyer Opatovski) (1886 Mława - I954 New York), Prosaschriftsteller, Kritiker, Übersetzer, besuchte eine russische Volksschule und erhielt eine jüdische Bildung im Elternhaus. Nach der Handelsschule in Warschau und einem begonnenen Ingenieurstudium in Nancy emigrierte er 1907 nach New York, wo er sich der Gruppe Di yunge anschloss. I9IO debütierte er mit der Erzählung "Afyener zayt brik«; seit I9I4 schrieb er regelmäßig Beiträge für Der tog. Mehrere Reisen nach Polen und Israel, außerdem in die Sowjetunion und nach Südamerika. Neben seinem Engagement in den jiddischen Kulturinstitutionen war er am Lehrerseminar tätig.

A shikhputser (S. 59). Erstdruck: Naye folkstsaytung (Warschau) IO (32I), I. II. I935: 7 .

Yom hadin (S. 63). Erstdruck: Yoysef Opatoshu und H. Leyvik (Hg.), 1939: Zamlbikher. Heft 4. New York: Farlag atlantik: 232-246.

Bibliographie (Auswahl): Khanukov, Leyvik, 1939: "Der dertseyler yoysef opatoshu «. In: Der hamer (New York) I2 (2): 66-68. Koller, Sabine, Gennady Estraikh und Mikhail Krutikov (Hg.), 2013: Joseph Opatoshu: A Yiddish Writer Between Europe and America. London: Legenda. Literarishe bleter (Warschau) 7 (6), 7.2.1930 - eine Opatoshu gewidmete Ausgabe. Mayzl, Nakhmen, 1957: "Yoysef opatoshu «. In: Noente un eygene. New York: Ikuf, 283-294. Pomerants, Gershn, 1938: "Yoysef opatoshus naye dertseylungen «. In: Literarishe bleter (Warschau) I5 (39-40), 21. IO. 1938: 663-664. 
Rokhl Oyerbakh (Rachel Auerbach) (1903 Lanowitz - 1976 Tel Aviv), Schriftstellerin, Historikerin, Essayistin, studierte nach Abschluss des Gymnasiums in Lemberg Philosophie, Psychologie und Geschichte. 1925 erste Veröffentlichungen in der polnischsprachigen Chwila, Ende der 1920er Jahre auch in der jiddischen Tageszeitung Nayer morgn. Seit 1933 in Warschau als Journalistin und Übersetzerin tätig, seit 1939 Mitarbeiterin von Dr. Emanuel Ringelblum und dem , Oyneg-Shabes-Archiv; 1950 emigrierte sie nach Israel. Als eine der ersten erkannte sie die Bedeutung von Zeitzeugenberichten für die historische Forschung und sammelte diese seit 1954 für Yad Vashem.

Teymener shkheynim (S. 13)

Di zuntikdike ,fayf-o-klokn bay froy tsetsilya slapak (S. 17)

Mangers, balader (S. 24)

Bibliographie (Auswahl): Auerbach, Rachel, 2008: "A Soup Kitchen in the Warsaw Ghetto: From the Memoirs of Rachel Auerbach " (übersetzt von Seymour Levitan). In: Bridges 13 (2): 96-97. Kassow, Samuel, 2015: "The Warsaw Ghetto in the Writings of Rachel Auerbach «. In: Dynner, Glenn und François Guesnet (Hg.): Warsaw. The Jewish Metropolis: Essays in Honor of the 75th Birthday of Professor Antony Polonsky. Leiden: Brill, 496-5I4. Mark, Feygl, 1975: "Hunger un toyt un mentsh «. In: Di goldene keyt (Tel Aviv) 86: 177-182. Gal-Ed, Efrat, 2016: „Rachel Auerbach«. In: Dies.: Niemandssprache. Itzik Manger - ein europäischer Dichter. Berlin: Jüdischer Verlag im Suhrkamp Verlag, 302-310. Roskies, David G. (Hg.), 2019: Voices from the Warsaw Ghetto. Writing Our History. New Haven, Conn.: Yale University Press. Szymaniak, Karolina, 20I8: „On the Ice Floe: Rachel Auerbach - The Life of a Yiddishist Intellectual in Early Twentieth Century Poland «. In: Laczó, Ferenc und Joachim von Puttkammer (Hg.): Catastrophe and Utopia. Jewish Intellectuals in Central and Eastern Europe in the I93os and I940s. Berlin: De Gruyter Oldenbourg, 304-352.

Roze Palatnik (1904 Kroshnik - 19[79]8I Rio de Janeiro), Prosaschriftstellerin, Journalistin, erhielt eine traditionell-jüdische Erziehung, erwarb im Selbststudium säkulares Wissen und umfassende Sprachkenntnisse. Zunächst als Lehrerin tätig gewann sie 1926 einen Literaturwettbewerb der Warschauer Zeitung Velt-shpigl mit dem Beitrag "Laydnshaft un oysgelasnkayt«. 1927 Emigration nach Frankreich, wo sie, als Sekretärin arbeitend, Erzählungen in der Handls-tsaytung veröffentlichte, bevor sie 1936 nach Rio de Janeiro übersiedelte. Dort verfasste sie zahlreiche Beiträge für die jiddische Presse Süd- und Nordamerikas.

Kaykelekh (S. 299)

Bibliographie (Auswahl): Cytrynowicz, Roney, 2018: "The Yiddish Side of Jewish Brazil«. In: Malena Chinski und Alan Astro (Hg.): Splendor, Decline, 
and Rediscovery of Yiddish in Latin America. Leiden, Boston: Brill, I5-4I. Fabel, Nachman, 2010: Literatura Ídiche no Brasil. São Paulo: Humanitas. Mayzl, Gitl, 1955: "Fun kroshnik biz rio-de-zhaneyro «. In: Parizer tsaytshrift (Paris) 3 (г) : 133-135. O. A., I96r: „Di novelistin roze palatnik«. In: Heymish (Tel Aviv) 5 (59-60): 17 .

Yitskhok-Leybush Perets (I852 Zamość - I915 Warschau), Dichter, Prosaund Theaterschriftsteller, Essayist, erhielt durch Privatlehrer eine jüdische Bildung und erwarb umfassende Sprachkenntnisse. I877 Veröffentlichung hebräischer Lyrik, I888 literarisches Debüt auf Jiddisch mit der Ballade "Monish". Seit I89I bis zu seinem Lebensende war der zuvor als Anwalt tätige Perets Angestellter der Jüdischen Gemeinde Warschau; seine Wohnung wurde zu einem literarischen Treffpunkt und er selbst zum Förderer unzähliger junger Schriftsteller.

Der meshulekh (S. 3II). Erstdruck: Bakante bilder. Warschau: Boymriter, I89o. Übersetzungen: "The Messenger « in: Perez, Isaac Loeb, 1906: Stories and Pictures. Philadelphia, Pa.: The Jewish Publication Society of America, IoI-II6 (übersetzt von Helena Frank); in: Peretz, I. L., 1927: Bontshe the Silent. Philadelphia, Pa.: David McKay, 37-48 (übersetzt von I. L. Rappoport); in: Peretz, I. L., 1958: In This World and the Next: Selected Writings of I. L. Peretz. New York, NY: Thomas Yoseloff, 176-185 (übersetzt von Moshe Spiegel).

Di toyte shtot (S. 319). Erstdruck: Die jiddische Fassung ist eine Selbstübersetzung der hebräischen Geschichte "Ir ha-metim « in: Ha-tsefira (Warschau), 5. und 7.8.1892. Übersetzungen: "The Dead Town « in: Howe, Irving und Eliezer Greenberg (Hg.), 1989: A Treasury of Yiddish Stories. New York, NY: Viking Press, 205-213 (übersetzt von Irving Howe); "The Dead Town « in: Wisse, Ruth (Hg.), 1990: The I. L. Peretz Reader. New York, NY: Schocken, I62-17I (übersetzt von Hillel Halkin).

Bibliographie (Auswahl): Molisak, Alina und Shoshana Ronen (Hg.), 2017: The Trilingual Literature of Polish Jews from Different Perspectives. In Memory of I. L. Peretz. Newcastle upon Tyne, UK: Cambridge Scholars Publishing. Blank, Inge und Heidi Hein, 20oI: »I. L. Peretz' Blick auf das sshtetk. Die Reportage über seine Provinzreise in historischer Perspektive ". In: Aschkenas II (2): 497-5I4. Caplan, Marc, 2007: "The Fragmentation of Narrative Perspective in Y. L. Peretz's > Bilder Fun a Provints-Rayze«". In: Jewish Social Studies I4 (I), (New Series): 63-88. Frieden, Ken, 1995: Classic Yiddish Fiction: Abramovitsh, Sholem Aleichem, and Peretz. New York, NY: SUNY Press. Miron, Dan, 1973: A Traveller Disguised: The Rise of Modern Yiddish Fiction in the Nineteenth Century. Syracuse, NY: Syracuse University Press. Niger, Samuel, 1952: Y.l.perets: zayn lebn, zayn firndike perzenlekbkayt, zayne hebreishe un yidishe shriftn, zayn virkung. Buenos Aires: Argentiner opteyl fun 
alveltlekhn yidishn kultur-kongres. Wisse, Ruth R., 199I: I. L. Peretz and the Making of Modern Jewish Culture. Seattle, WA: University of Washington Press.

Melekh Ravitsh (Melech Ravitch, Zekharye-Khone Bergner) (I893 Radymno - 1976 Montreal), Dichter, Essayist, Dramatiker, Übersetzer und Kulturaktivist, erhielt neben einer säkularen eine traditionell-jüdische Bildung; I9IO veröffentlichte er erste Gedichte in Der yudisher arbeyter. Seit I92I in Warschau, Sekretär des jiddischen Schriftstellerverbandes (Tłomackie I3); Mitbegründer der Gruppe Khalyastre und der Zeitschrift Literarishe bleter. Während der I930er Jahre in Australien, Nord- und Südamerika sowie Israel lebend, emigrierte er 1956 nach Montreal, wo er u. a. seine Memoiren Dos mayse-bukh fun mayn lebn verfasste.

Di fatsyate (S. 423)

Der shklafnmark (S. 429)

Bibliographie (Auswahl): Melech Rawitsch, 1996: Das Geschichtenbuch meines Lebens. Salzburg (u. a.): Müller, herausgegeben und übersetzt von Armin Eidherr. Gal-Ed, Efrat, 2016: "Melech Ravitch «. In: Dies.: Niemandssprache. Itzik Manger - ein europäischer Dichter. Berlin: Jüdischer Verlag im Suhrkamp Verlag, 254-270. Korn, Rokhl, 1976: "An instants far zikh aleyn. Tsum ondenk fun melekh ravitsh «. In: Di goldene keyt (Tel Aviv) 9r: 22-25. Margolis, Rebecca, 2016: "Remaining Alive in Silence? Melekh Ravitch as Yiddish Catalyst; Montreal, 194I-54". In: East European Jewish Affairs 46 (2): 192-209. Ruta, Magdalena, 20II: "'Tłomacke 13، in Erinnerung von Schriftstellern und Journalisten jiddischer Sprache«. In: Convivum: II-32. Yeshurin, Yefim und V. Ostreger, 1954: Melekh ravitsh bibliografye. Montreal: M. Ravitch Book Committee at the Jewish Public Library.

Avrom Reyzen (Reisen) (1876 Koydanov - 1953 New York), Dichter, Prosaschriftsteller, Übersetzer, Publizist, erhielt neben einer jüdischen auch eine säkulare Bildung. Erste Artikel in der Petersburger jiddischen Presse, I89I Veröffentlichung seines Gedichts "Ven dos lebn iz farbitert " in Y. L. Peretz's Di yudishe bibliotek. Seit 1899 in Warschau trat Reyzen nicht nur als Autor, sondern vermehrt auch als Herausgeber in Erscheinung und gründete 1902 den Verlag "Tsentral «. I9II emigrierte er in die USA, wo er Herausgeber literarischer Zeitschriften und langjähriger Mitarbeiter von Forverts, Frayhayt und Di tsukunft wurde.

Di naye shayle (S. 45I). Erstdruck: Der tsayt-gayst (New York) I (2), I.9.1905. Übersetzung: "The New Question « in: Reisen, Abraham, 1992: The HeartStirring Sermon and Other Stories. Woodstock, NY: The Overlook Press, I43-I5O (übersetzt von Curt Leviant). 
A shtetl on vayber (S. 457). Erstdruck: Haynt 5 (234), I2. [25.] IO. 1912: 2.

Bibliographie (Auswahl): Mayzl, Nakhmen, 1957: „Avrom reyzen «. In: Noente un eygene. New-York: Ikuf, I28-I44. Sitarz, Magdalena, 2007: »Im Kreis der Schüler von Perets: die frühen Novellen von Avrom Reyzen «. In: Scripta Judaica Cracoviensia 5: 77-85. Shneyfal, Sh. K., 1908: „Avrom reyzens noveles. A kritisher iberblik «. In: Di tsukunft (New York) I3 (9): 540 - 544. Shpitalnik, L., 1936: Avrom reyzen. Zayn lebn un shafn. New York: Sholem aleykhem folksinstitut. Slutski, Shloyme, 1956: Avrom reyzen-bibliografye. New York: Bibliotek un arkhiv baym yidishn lerer seminar un folksuniverzitet.

Leyb Rokhman (I9ı8 Mińsk Mazowiecki - I978 Jerusalem), Erzähler, Journalist, erhielt neben einer traditionell-jüdischen Bildung auch Unterricht in weltlichen Fächern; erste journalistische Erfahrungen beim Varshever radio. Nach der Flucht aus einem Arbeitslager überlebte er den Zweiten Weltkrieg im Versteck bei einer polnischen Dorfbewohnerin. 1945-1948 lebte Rokhman in der Schweiz. 1950 emigrierte er nach Israel und wurde Mitarbeiter verschiedener Radioprogramme und Zeitungen. Sein erstes literarisches Werk, Un in dayn blut zolstu lebn, erschien 1949 in Paris; 1978 veröffentlichte er den Erzählungsband Der mabl.

Dos rod (S. 439)

Bibliographie (Auswahl): Apelfeld, Arn, 1979: "Yedid-nefesh. Vegn leyb rokhman «. In: Di goldene keyt (Tel Aviv) 99: 15-20. Miriam, Rivke, 1984: »Mayn foter«. In: Di goldene keyt (Tel Aviv) II4: I03. Sfard, Dovid, 1972: "Di khurbn-teme nusekh leyb rokhman". In: Di goldene keyt (Tel Aviv) 75: 230-236. Yanosovitsh, Yitskhok, 1988: „Der shrayber un mentsh leyb rokhman «. In: Yerusholaymer almanakh (Jerusalem) I8: I7I-I77.

Zusman Segalovitsh (I884 Białystok - 1949 New York), Dichter, Prosaschriftsteller, besuchte den Cheder und erhielt zusätzlich Privatunterricht. Veröffentlichung eines russischen Gedichts 1903, Debüt auf Jiddisch in der Petersburger Tageszeitung Der fraynd 1904. Seit 1919 in Warschau lebend wurde er u. a. Mitarbeiter von Haynt, 1929 von Der moment und engagierte sich im jiddischen Schriftstellerverband. Erinnerungen an jene Zeit hielt er in dem Band Tlomatske I3 fest; von 1928 bis 1930 leitete er die Warschauer Abteilung des P.E.N.-Clubs. 1939 floh er aus Warschau und gelangte über Umwege 194I nach Israel.

Panna zofia (S. 289). Erstdruck: Haynt (Warschau) 20 (27I), 2. I2. 1927: 6 (mit erheblichen Abweichungen).

Der groyser yon-tev (S. 293). Erstdruck: "A groyser yontev«. In: Der moment (Warschau) I9 (290), I4. I2. 1928: 6. 
Bibliographie (Auswahl): Fareyn fun yidishe literatn un zhurnalistn in varshe (Hg.), I933: Vegn z. segalovitsh. Tsu zayn draysik yerikn shrayberyoyvl, 1903-1933. Warschau: Farlag kh. bzhoza. Kazdan, Khayim-Shloyme, 1979: Zusman segalovitsh: (a monografye). New York: Unzer tsayt. Mayzl, Nakhmen, 1957: "Z. segalovitsh «. In: Noente un eygene. New-York: Ikuf, 183-194. Tenenboym, Shye, 1981: ") Ikh gey tsu mayne farbrente yidn ‘. Zusman segalovitsh - der mekoynen fun poylishn yidntum«. In: Di tsukunft (New York) 87 (4): I47-I5I.

Lamed Shapiro (Levi-Yehoyshue Shapiro) (1878 Rzhyshtshev - 1948 Los Angeles), Erzähler, Dichter, Essayist, erhielt neben der jüdischen auch eine säkulare Bildung. Zunächst Jiddisch, Hebräisch und Russisch schreibend debütierte er 1903 mit der Erzählung "Di fligl in Sukes-blat. Infolge der Pogrome emigrierte er 1905 über London in die USA, wo er für jiddische Zeitungen schrieb. Nach einem kurzen Aufenthalt in Warschau I9Io Rückkehr in die USA, wo er weiterhin für die jiddische Presse tätig war; seit 1920 Mitherausgeber der kommunistischen Zeitschrift Funken; 1934 Herausgeber der literarischen Zeitschrift Studio. Daneben galt Shapiros Interesse der Farbphotographie, der er sich mehrere Jahre in seinem Labor in Los Angeles widmete.

Roykh (S. 46I). Erstdruck: Velt-ayn velt-oys (zamlbukh). New York: Amerika, I916, 33-42. Übersetzungen: "Smoke« in: Howe, Irving und Eliezer Greenberg (Hg., Übers.), 1989: A Treasury of Yiddish Stories. New York, NY: Viking Press, 334-339; "Smoke" in: Shapiro, Lamed, 2007: The Cross and Other Jewish Stories. Hg. von Leah Garrett. New Haven, Conn. (u. a.): Yale University Press, IOI-IO7 (übersetzt von Irving Howe und Eliezer Greenberg).

Nyu-yorkish (S. 468). Erstdruck: Vokh (New York), Nr. 20, I4. Februar 1930: 8-I2. Übersetzungen: "New Yorkish « in: Shapiro, Lamed, 2007: The Cross and Other Jewish Stories. Hg. von Leah Garrett. New Haven, Conn.: Yale University Press, 198-212 (übersetzt von Lawrence Rosenwald).

Bibliographie (Auswahl): Garrett, Leah, 20Ir: "Dazed and Confused: Lamed Shapiro's American Stories«. In: Studies in American Jewish Literature 30: 47-59. Rivkin, Borekh, 1931: "L. shapiro - der letster fun di rishoynim. `Nyu-yorkish un andere zakhn ‘ fun 1. shapiro«. In: Di tsukunft (New York) 36 (6): 424-429. Shapiro, Lamed, 1945: Der shrayber geyt in kheyder. Los Angeles: Aleyn. Szwarcman-Czarnota, Bella, 2017: "The Two Facets of Lamed Shapiro and Isaac Leib Peretz«. In: Molisak, Alina und Shoshana Ronen (Hg.): The Trilingual Literature of Polish Jews from Different Perspectives. In Memory of I. L. Peretz. Newcastle upon Tyne: Cambridge Scholars Publishing, 57-74. Wolf, Robert Harvey, 1994: A Yiddish Manichaean: The Dualistic Fiction of Lamed Shapiro. New York, NY: Columbia University. 
Sholem-Aleykhem (Sholem Aleichem, Scholem Alejchem; Sholem-Yankev Rabinovitsh) (I859 Pereyaslav - I916 New York), Prosaschriftsteller, Dramatiker; Besuch des Cheder sowie einer russischen Schule; nach ersten Veröffentlichungen auf Hebräisch erschien 1883 die jiddische Novelle "Tsvey shteyner ". Zunächst u.a. als Hauslehrer und Kronrabbiner tätig widmete sich Sholem-Aleykhem in den folgenden Jahrzehnten zunehmend seiner schriftstellerischen Arbeit und gründete I888 die erste jiddische literarische Zeitschrift Di yidishe folks-bibliotek. In den I89oer Jahren entstanden die ersten Teile von Menakhem-Mendl und Tevye der milkhiker, an deren Fortsetzungen er in den nächsten Jahrzehnten arbeitete. Nach Aufenthalten in Odessa, Lemberg und Genf gelangte er 1906 über London erstmals in die USA, kehrte ein Jahr später nach Europa zurück und ließ sich I9I4 endgültig in New York nieder.

Khabne (S. 493). Erstdruck: Di yudishe tsukunft (London-Paris-Krakau-New York, 1904-1906) (2), 1905: 108- II0. Übersetzungen: "The Village of Habne" in: Sholem Aleichem, 1966: Old Country Tales. Hg. und übers. von Curt Leviant. New York, NY: Putnam; "Chabne« in: Scholem Alejchem, 1997: 75000 und andere Geschichten um Gott, Geld und Glück. Hg. und übers. von Dan Wiener. Leipzig: Reclam, Ioo-II5; "Chabne " in: Sholem Aleichem, 1998: Nineteen to the Dozen. Hg. von Ken Frieden. Syracuse, NY: Syracuse University Press, I03-II4 (übersetzt von Ted Gorelick).

A frier peysekh (S. 502). Erstdruck: Der fraynd (Petersburg) 6 (74), 28.3. [10. 4.] 1908: 2; (75) 30.3. [12. 4.] 1908: 2; (76) 31. 3. [13. 4.] 1908: 2 f. Übersetzungen: An Early Passover: The Plight of a Russian Emigrant, or, The Mystery of the Jewish Calendar. New York, NY: Clifton Pub. Co., 1966 (übersetzt von George Zinberg).

Bibliographie (Auswahl): Erlich, Victor, 1964: "A Note on the Monologue As a Literary Form: Sholem Aleichem's Monologn; A Test Case «. In: Dawidowicz, Lucy S. (Hg.): For Max Weinreich on His Seventieth Birthday: Studies in Jewish Languages, Literature, and Society. London (u.a.): Mouton, 44-5o. Estraikh, Gennady, Jordan Finkin, Kirstin Hoge und Mikhail Krutikov (Hg.), 20I2: Translating Sholem Aleichem: History, Politics and Art. London: Legenda. Frieden, Ken, 1989: "Sholem Aleichem: Monologues of Mastery«. In: Modern Language Studies I9 (2): 25-37. Mantovan, Daniela, 2012: "Writing and Speech in Sholem Aleichem's Monologues". In: Heil, Johannes und Daniel Krochmalnik (Hg.): Jüdische Studien als Disziplindie Disziplinen der Jüdischen Studien. Heidelberg: Winter, 30I-310. Miron, Dan, 20I9: "Reise ins Zwielicht. Zu Scholem Alejchems Eisenbahngeschichten «. In: Gal-Ed, Efrat, Gernot Jonas und Simon Neuberg (Hg.): Scholem Alejchem: Eisenbahngeschichten. Schriften eines Handlungsreisenden. Berlin: düsseldorf university press, 365-442 (übersetzt von Liliane Meilinger). 
Fradl Shtok (I89o Skale - I952 Hollywood), Dichterin, Erzählerin, bereits als Schülerin mit der deutschen Literatur vertraut, emigrierte sie 1907 in die USA. Nach der Veröffentlichung ihres ersten Gedichts "Serenade" I9IO publizierte Shtok in den folgenden Jahren in literarischen Zeitschriften und Anthologien, stand in Kontakt mit der das Konzept des l'art pour l'art propagierenden Gruppe Di yunge und führte als eine der ersten das Sonett in die jiddische Dichtung ein. Ihr I9I9 erschienener Band Gezamelte ertsehlungen erhielt neben sehr positiven auch negative Kritiken, woraufhin sie sich der englischen Sprache zuwandte. 1927 veröffentlichte sie den Roman Musicians Only.

Fridrikh shiler (S. 483)

A rede (S. 488)

Bibliographie (Auswahl): Fuks, Avrom-Moyshe, I920: "Vegn fradl shtoks bukh «. In: Kritik (Wien) I (6), 25. 12.1920: 29f. Gollance, Sonia, 2017: "A Dance. Fradel Shtok Reconsidered «. In: In geveb: A Journal of Yiddish Studies. <https://ingeveb.org/articles/a-dance-fradel-shtok-reconsidered>, I2. 10. 2020. Niger, Shmuel, 1920: "Di ertseylungen fun fradl shtok«. In: Di tsukunft (New York) 25 (I0): 608-6ro. Olgin, 1920: »Pesimizm. Gezamelte ertsehlungen fun fradel shtok «. In: Di naye velt (New York) 7 (265), 9. I. 1920: I6f. Ravitsh, Melekh, I923: ")Gezamelte ertsehlungen ‘ fun fradel shtok «. In: Bikher-velt (Warschau) 2 (I-2), 1923: 64-66. Zaydenfeld, Dovid, 1920: "A marionetn-molerin: vegn fradl shtoks bukh ertsehlungen «. In: Renesans (London) 2 (2), Mai 1920: 150-152.

Itshe-Meyer Vaysenberg (I88I Żelechów - 1938 Legionowo), jiddischer Prosaschriftsteller, erhielt eine traditionell-jüdische Bildung im Cheder; 1904 Veröffentlichung seiner ersten beiden Kurzgeschichten "Der kitl " und "Dor hoylekh vedor bo " in Perets' Di yudishe bibliotek; zwei Jahre später erschien die Novelle $A$ shtetl. Unter den jiddischen Schriftstellern Polens nahm Vaysenberg teilweise eine oppositionelle Haltung ein; so forderte er u.a., dass sich sein jiddischer Dialekt in einer entsprechenden Rechtschreibung widerspiegele. Außer als Verfasser zahlreicher Erzählungen trat Vaysenberg in den folgenden Jahren als Herausgeber jiddischer Zeitschriften und Förderer junger Autoren in Erscheinung.

Di meshugene in dorf (S. I8I). Erstdruck: Dos yudishe vort (Krakau) I (6), 7.2.1905: 3-7; (7), I4. 2. 1905: 5-7; (8), 2I. 2. 1905: 4-7.

Mazl-tov (S. 199). Erstdruck: Der veg (Warschau) (88), 5. I. 1906: 2.

Übersetzungen: "Mazel Tov « in: Howe, Irving und Eliezer Greenberg (Hg., Übers.), 1989: A Treasury of Yiddish Stories. New York, NY: Viking Press, $295-296$. 
Bibliographie (Auswahl): Botoshanski, Yankev, I952: "I. m. vaysenberg «. In: Pshat (peyrushim oyf yidishe shrayber). Buenos Aires, 69-92. Sherman, Joseph, 2007: "Naturalism and Itsik Meir Weissenberg (I878-1938)«. In: Midstream 53 (4): I6f. Vinogura, Borekh, 1932: "I. m. vaysenberg un zayn shafung ". In: Vokhnshrift far literatur, kunst un kultur (Warschau) 2 (IO), 4.3.1932: 3f.; (II), II.3.1932: 4f; (I2), I8.3.1932: 5; (I3), 25.3.1932: 4. Weissenberg-Akselrod, Pearl, I986: Y. m. vaysenberg. Zayn leben un shafn, 1878-1938. Montreal: Y. m. vaysenberg bukhfond.

Yisroel-Yoyshue Zinger (Israel Joshua Singer) (I893 Biłgoraj - I944 New York), Prosaschriftsteller, Journalist. Der Bruder von Ester Kreytman und Yitskhok Bashevis Zinger erhielt eine traditionell-jüdische Bildung, erwarb jedoch durch heimliche Lektüre auch säkulare Literaturkenntnisse. I9I8 Veröffentlichung seiner ersten Erzählung in einem orthodoxen Wochenblatt, seit I92I Mitglied der expressionistischen Gruppe Khalyastre in Warschau, Korrespondent des Forverts (New York). I934 emigrierte Zinger in die USA, wo er nicht nur als Romanautor (Di brider ashkenazi, Di mishpokhe karnovski), sondern auch als Essayist in Erscheinung trat.

Professor arkadi gritshendler (S. 203). Erstdruck: Forverts (New York) XXV (9250), II. 3. 1923: I9 (mit Abweichungen).

A tragedye tsulib dem, vos me hot in himl farbitn di yoytsres (S. 2I4). Erstdruck: Unter dem Titel »Emese pasirungen « in: Forverts (New York) XLVII (I6935), 29. 4. 1944: 2, 7. Übersetzungen: Singer, Israel Joshua, 1970: Of a World That Is No More. New York, NY: Vanguard Press (übersetzt von Joseph Singer); Singer, Israel Joshua, 1993: Von einer Welt, die nicht mehr ist: Erinnerungen. Ungekürzte Ausgabe. Frankfurt am Main: Fischer-Taschenbuch-Verlag (übersetzt von Gertrud Baruch).

Bibliographie (Auswahl): Fuks, [Avrom-]M[oyshe], 1925: "Y. y. zinger«. In: Literarishe bleter (Warschau) 2 (79), 6. II. 1925: 214-216. Howe, Irving, 1966: " The Other Singer «. In: Commentary 43, Nr. I (März): 78 -82. Mayzl, Nakhmen, 1926: "Y. y. zinger". In: Literarishe bleter (Warschau) 2 (I3I), 5.11. 1926: 728f. Norich, Anita, 1990: "The Family Singer and the Autobiographical Imagination «. In: Prooftexts Io (I): 91-107. Dies., 1991: The Homeless Imagination in the Fiction of Israel Joshua Singer. Bloomington, Ind.: Indiana University Press. 